Homology, Homotopy and Applications, vol.11(1), 2009, pp.185-202

\title{
MODELS AND VAN KAMPEN THEOREMS FOR DIRECTED HOMOTOPY THEORY
}

\author{
PETER BUBENIK
}

\author{
(communicated by Ronald Brown)
}

\begin{abstract}
We study topological spaces with a distinguished set of paths, called directed paths. Since these directed paths are generally not reversible, the directed homotopy classes of directed paths do not assemble into a groupoid, and there is no direct ana$\log$ of the fundamental group. However, they do assemble into a category, called the fundamental category. We define models of the fundamental category, such as the fundamental bipartite graph, and minimal extremal models which are shown to generalize the fundamental group. In addition, we prove van Kampen theorems for subcategories, retracts, and models of the fundamental category.
\end{abstract}

\section{Introduction}

\subsection{Directed spaces and directed homotopies}

The field of directed algebraic topology studies directed spaces. That is, topological spaces together with a (local) order, or more generally, spaces together with a subset of allowed paths, called directed paths. In either approach, the directed paths are generally not reversible. Consequently, the directed homotopy classes of directed paths behave much differently from the usual homotopy classes of paths (see Example 1.3). As many topologists are unfamiliar with directed algebraic topology, we give a leisurely introduction, which includes the main new constructions and results of this paper.

A motivation for this study comes from the field of concurrent (parallel) computing, in which multiple processes have access to shared resources. A directed space models the state space of such a system, and the directed paths model the execution paths. General relativity provides another possible application. For more details, the reader is referred to the papers $[\mathbf{9}, \mathbf{1 4}]$.

A number of categorical settings have been used to develop directed algebraic topology. These include partially ordered spaces (pospaces) $[\mathbf{4}, \mathbf{6}]$, local pospaces $[\mathbf{5}, \mathbf{9}, \mathbf{2 3}]$,

Received February 14, 2009, revised March 18, 2009; published on May 21, 2009.

2000 Mathematics Subject Classification: Primary 55P99, 68Q85; Secondary 18A40, 18A30, 55U99.

Key words and phrases: directed homotopy, fundamental category, van Kampen theorem, d-space, reflective subcategory, coreflective subcategory, past retract, future retract, extremal model, fundamental bipartite graph.

This article is available at http://intlpress.com/HHA/v11/n1/a9

Copyright (C) 2009, International Press. Permission to copy for private use granted. 
preordered spaces [16], local preordered spaces [19], d-spaces [15, 21], flows [10], and cubical complexes (also known as higher-dimensional automata) [7, 8, 11, 20]. Here we work in the general setting of Grandis' $d$-spaces.

Definition $1.1([\mathbf{1 5}])$. A $d$-space is a topological space $X$ together with a set $d X$ of paths $\gamma:[0,1] \rightarrow X$, called directed paths or dipaths satisfying the following axioms:

1. for all $x \in X$, the constant path $c_{x}(t)=x$ is in $d X$,

2. $d X$ is closed under reparametrization: if $\gamma \in d X$ and $f:[0,1] \rightarrow[0,1]$ is continuous and non-decreasing then $\gamma \circ f \in d X$, and

3. $d X$ is closed under concatenation: if $\gamma_{1}, \gamma_{2} \in d X$ and $\gamma_{1}(1)=\gamma_{2}(0)$, then $\gamma \in d X$ where $\gamma(t)=\gamma_{1}(2 t)$, for $0 \leqslant t \leqslant \frac{1}{2}$, and $\gamma(t)=\gamma_{2}(2 t-1)$, for $\frac{1}{2} \leqslant t \leqslant 1$.

Since $f:[0,1] \rightarrow[0,1]$ in $(2)$ above need not be onto, subpaths of dipaths are also dipaths. A morphism of d-spaces $f: X \rightarrow Y$, called a dimap, is a continuous map which preserves dipaths. That is, $f(d X) \subseteq d Y$, where $f(\gamma)=f \circ \gamma$.

\section{Example 1.2.}

- Any topological space $X$ is a d-space with $d X$ equal to the set of all paths in $X$.

- Let $\vec{I}=(I, d I)$ where $I$ is the closed interval $[0,1]$ and $d I$ is the set of all nondecreasing continuous maps $I \rightarrow I$. Dipaths in d-space $X$ coincide with dimaps $\vec{I} \rightarrow X$.

- Let $\vec{S}^{1}$ be the unit circle together with all counterclockwise paths.

- Given two d-spaces $X$ and $Y$, then $X \times Y$ is a d-space with

$$
d(X \times Y)=d X \times d Y \quad \text { where } \quad(f, g)(t)=(f(t), g(t)) .
$$

- If $X$ is a d-space and $A \subseteq X$, then $A$ is a d-space with $d A$ equal to the subset of paths in $d X$ whose image is in $A$.

An advantage of using d-spaces over the more commonly used preordered spaces is that we can model loops, such as with $\vec{S}^{1}$.

A d-homotopy between dimaps $f, g: X \rightarrow Y$ is a dimap $H: X \times \vec{I} \rightarrow Y$ such that for all $x \in X, H(x, 0)=f(x)$, and $H(x, 1)=g(x)$. We write $H: f \stackrel{\simeq}{\longrightarrow} g$ and $H_{0}=f$ and $H_{1}=g$. Notice that this notion is not symmetric. To obtain an equivalence relation we take the transitive symmetric closure and say that $f$ is d-homotopic to $g$ if they are linked by a chain of d-homotopies, $f \stackrel{\simeq}{\longrightarrow} f_{1} \stackrel{\simeq}{\longleftarrow} f_{2} \stackrel{\simeq}{\longrightarrow} \cdots \stackrel{\simeq}{\longrightarrow} g$.

Given a dipath $\gamma$ from $a$ to $b$, let $[\gamma]$ denote the equivalence class of dimaps $\gamma^{\prime}: \vec{I}$ $\rightarrow X$ with $\gamma^{\prime}(0)=a, \gamma^{\prime}(1)=b$ and $\gamma^{\prime}$ d-homotopic to $\gamma$ relative to $\{a, b\}$. That is, we insist that the d-homotopies linking $\gamma^{\prime}$ and $\gamma$ leave the endpoints fixed. Call this a directed homotopy class of directed paths from $a$ to $b$, or more simply, a homotopy class of dipaths.

Example 1.3. The directed paths up to directed homotopy of a d-space are very different from the paths up to homotopy of the underlying topological space.

For example, an undirected path $\gamma: I \rightarrow X$ need not be homotopic relative to its endpoints to a directed path in a d-space $X$. Consider the following example, which is a subspace obtained from $\vec{I} \times \vec{I}$ by removing two squares. 


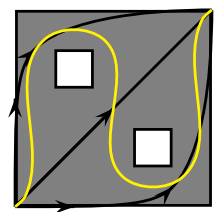

Furthermore, directed paths in a space that is contractible in the undirected sense are not necessarily d-homotopic. In the following figure we have two non-homotopic dipaths in a contractible d-space obtained from $\vec{I} \times \vec{I} \times \vec{I}$ by removing two isothetic parallelepipeds which intersect the boundary of $X$.

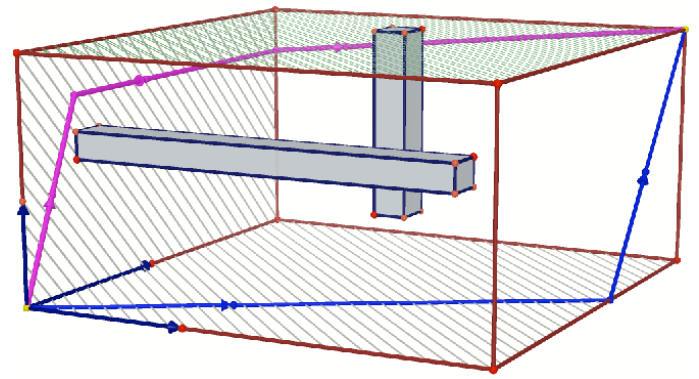

\subsection{The fundamental category}

In trying to understand the directed paths in a directed space, $X$, a basic object of study is the fundamental category, $\vec{\pi}_{1}(X)$. Its objects are the points in $X$, and for $a, b \in X$, the morphisms $\vec{\pi}_{1}(X)(a, b)$ are given by the directed homotopy classes of directed paths from $a$ to $b$. The undirected version of this definition results in the fundamental groupoid, in which all morphisms are invertible. When $X$ is a d-space the only invertible morphisms in the fundamental category are the homotopy classes for reversible dipaths.

In usual undirected algebraic topology, the fundamental groupoid is often simplified to the fundamental group by identifying the isomorphism classes of objects. That is, the fundamental group is the skeleton of the fundamental groupoid. However, for d-spaces where the only reversible paths are the constant paths, the fundamental category is its own skeleton.

This is a central difficulty, and has led to considerable research in directed algebraic topology. The goal is to reduce the fundamental category, which typically has uncountably many objects, to some considerably smaller and preferably finite structure that still contains 'the essential information'.

One approach, explored by Fajstrup, Goubault, Haucourt, and Raussen $[\mathbf{6 , ~ 1 3 , ~ 1 8 ] , ~}$ is to use the calculus of fractions or generalized equivalences to reduce the fundamental category to its component category. Here we follow Grandis' approach [16, 17] and look for a (possibly finite) full subcategory of the fundamental category that will provide an adequate model of the fundamental category.

Now we introduce some new notation that will be useful. Similar notation has been used for fundamental groupoids.

Notation 1.4. Let $\iota: A \hookrightarrow X$ be the inclusion of a subspace. Let $\vec{\pi}_{1}(X, A)$ denote the full subcategory of $\vec{\pi}_{1}(X)$ generated by $A$. That is, $\vec{\pi}_{1}(X, A)$ has as objects the 
points in $A$, and for $a, b \in A, \vec{\pi}_{1}(X, A)(a, b)=\vec{\pi}_{1}(X)(a, b)$. Let $\vec{\pi}_{1}(\iota): \vec{\pi}_{1}(X, A) \rightarrow$ $\vec{\pi}_{1}(X)$ denote the inclusion. For $x \in X$ we simplify $\vec{\pi}_{1}(X,\{x\})$ to $\vec{\pi}_{1}(X, x)$.

\subsection{Fundamental bipartite graphs}

We introduce a new full subcategory of the fundamental category that is useful for many of the d-spaces that appear in applications.

Definition 1.5. The objects of a category $\mathbf{C}$ have a preorder defined by $x \leqslant y$ if and only if there exists a morphism from $x$ to $y$. Call an object $a \in \mathbf{C}$ minimal if $x \leqslant a$ implies $x=a$. Similarly, define $b \in \mathbf{C}$ to be maximal if $b \leqslant x$ implies $b=x$. Say that an object is extremal if it is either maximal or minimal. Let $\operatorname{Extrl}(\mathbf{C})$ denote the set of all extremal objects in C. For a d-space $X$ we will sometimes let $\operatorname{Extrl}(X)$ denote $\operatorname{Extrl}\left(\vec{\pi}_{1}(X)\right)$. Define the fundamental bipartite graph of $X$ to be $\vec{\pi}_{1}(X, \operatorname{Extrl}(X))$. To view this category as a bipartite graph, we ignore the identity maps.

Example 1.6. Let $X$ be the subspace of $\vec{I} \times \vec{I}$ in the left-hand figure. Its fundamental bipartite graph has two vertices and four edges. We remark that the branching information is lost in this graph.
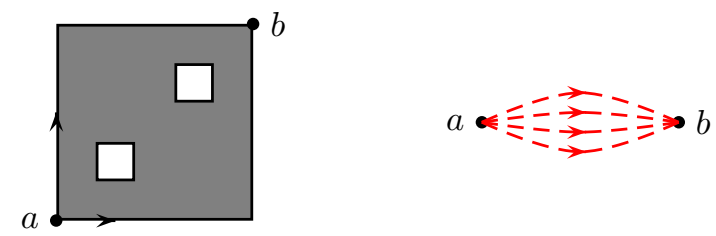

\subsection{Past retracts and future retracts}

In order to simplify the fundamental category, one obvious approach to the homotopy theorist is to apply directed homotopies to the underlying space.

Definition 1.7. Call a directed map $H: X \times \vec{I} \rightarrow X$ a future homotopy flow if $H_{0}=$ $\operatorname{Id}_{X}$ and a past homotopy flow if $H_{1}=\operatorname{Id}_{X}$. For a future (past) homotopy flow let $f$ equal $H_{1}\left(H_{0}\right)$. A future (past) homotopy flow induces a functor

$$
\vec{\pi}_{1}(X) \rightarrow \vec{\pi}_{1}(\operatorname{im} f) \cong \vec{\pi}_{1}(X, \operatorname{im} f) .
$$

Raussen $[\mathbf{2 1}]$ has carefully studied these flows.

A fruitful generalization at the level of the fundamental category is given by the following definition. In Section 2 we will see that our definition is equivalent to the categorical definition given by Grandis $[\mathbf{1 6}]$.

Definition 1.8. A future retract of $\vec{\pi}_{1}(X)$ is a subspace $A \subseteq X$ together with a homotopy class of dipaths $\left[\gamma_{x}\right]$ for all $x \in X$, with $\gamma_{x}(0)=x$ and $\gamma_{x}(1)=: x^{+} \in A$ such that for all homotopy classes of dipaths $[\gamma]: x \rightarrow a$ where $a \in A$, there is a unique morphism making the following diagram commute.

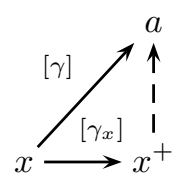

We also insist that for $a \in A,\left[\gamma_{a}\right]=\left[\operatorname{Id}_{a}\right]$. 
Example 1.9. In this example we describe a future retract of the square annulus, a subspace of $\vec{I} \times \vec{I}$. For all the points $x$ in the lower-left square, $x^{+}=a$ and for the remaining points $y, y^{+}=b$. So $A=\{a, b\}$. We can think of the future retract as pushing points forward in time in a way so that no decisions are made with respect to the future.

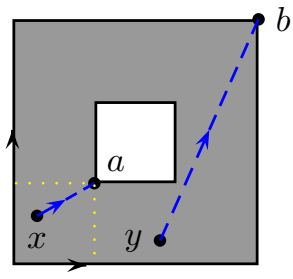

We should not be unduly concerned that these retracts are not induced by continuous maps. For in the classical undirected case, the skeleton functor from the fundamental groupoid of $S^{1}$ to $\pi_{1}\left(S^{1}\right)$ is not induced by a continuous map.

We remark that the definition implies that there is a unique morphism making the following diagram commute.

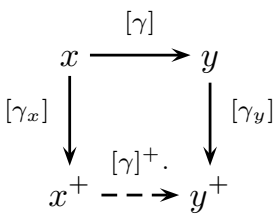

By uniqueness, $\left[\operatorname{Id}_{x}\right]^{+}=\left[\operatorname{Id}_{x^{+}}\right]$and $[\beta \circ \alpha]^{+}=[\beta]^{+} \circ[\alpha]^{+}$. That is, we have a functor $P^{+}: \vec{\pi}_{1}(X) \rightarrow \vec{\pi}_{1}(X, A)$. Also note that $P^{+} \vec{\pi}_{1}(\iota)=\operatorname{Id}_{\vec{\pi}_{1}(X, A)}$.

Dually, one has past retracts, which induce a functor $P^{-}: \vec{\pi}_{1}(X) \rightarrow \vec{\pi}_{1}(X, A)$. For an explicit definition, see Definition 2.2.

\subsection{Extremal models}

Just as we took the transitive symmetric closure of d-homotopies, we are led to consider chains of past and future retracts. In Definition 2.7, we will generalize our previous definitions of future retracts and past retracts to full subcategories of the fundamental category. This allows us to define the following new model of a d-space $X$.

Definition 1.10. An extremal model of $X$ is a chain of future retracts and past retracts

$$
\vec{\pi}_{1}(X) \stackrel{P_{1}^{+}}{\longrightarrow} \vec{\pi}_{1}\left(X, X_{1}\right) \stackrel{P_{2}^{-}}{\longrightarrow} \vec{\pi}_{1}\left(X, X_{2}\right) \stackrel{P_{3}^{+}}{\longrightarrow} \cdots \stackrel{P_{n}^{ \pm}}{\longrightarrow} \vec{\pi}_{1}(X, A),
$$

(where $A \subseteq \cdots \subseteq X_{2} \subseteq X_{1} \subseteq X$ ) such that $\operatorname{Extrl}(X) \subseteq A$. Call an extremal model minimal if there are no non-trivial future or past retracts $\vec{\pi}_{1}(X, A) \rightarrow \vec{\pi}_{1}\left(X, A^{\prime}\right)$ such that $\operatorname{Extrl}(X) \subseteq A^{\prime}$

Example 1.11. Let $X$ be a non-empty path-connected topological space. Let $d X$ be the set of all paths in $X$ and choose $x \in X$. Then $\vec{\pi}_{1}(X)$ is the fundamental groupoid, and $\vec{\pi}_{1}(X, x)$ is the fundamental group. If $X=\{x\}$ then $\operatorname{Extrl}(X)=\{x\}$, but otherwise $\operatorname{Extrl}(X)$ is empty. Set $\left[\gamma_{x}\right]=\left[\operatorname{Id}_{x}\right]$ and for all other $y \in X$ choose a homotopy 
class $\left[\gamma_{y}\right]$ of paths from $y$ to $x$. This induces a functor $\vec{\pi}_{1}(X) \rightarrow \vec{\pi}_{1}(X, x)$ which is the skeleton functor, a future retract, a past retract, and a minimal extremal model.

Example 1.12. Here we give three examples of an extremal model obtained from a future retract followed by a past retract. In each case, we have included the generating non-identity morphisms in the final figure.

1. The square annulus:
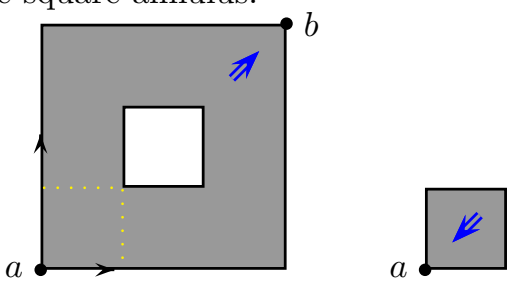

- $b$

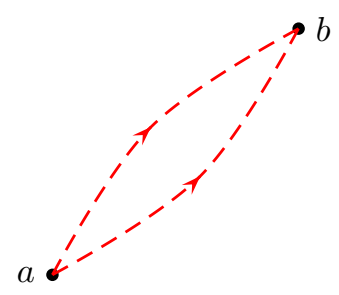

2. The Swiss flag:

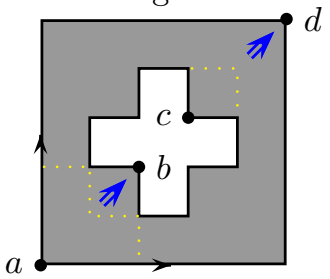

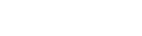

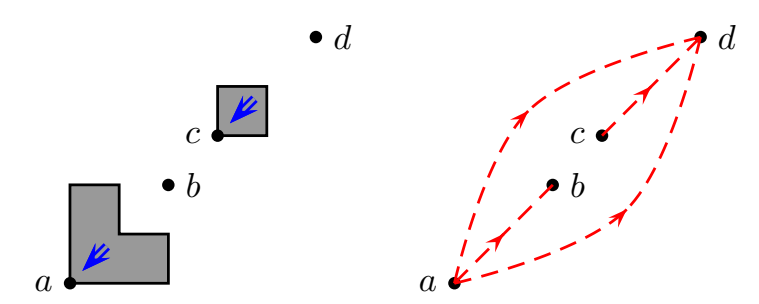

3. The directed square with two holes in series:
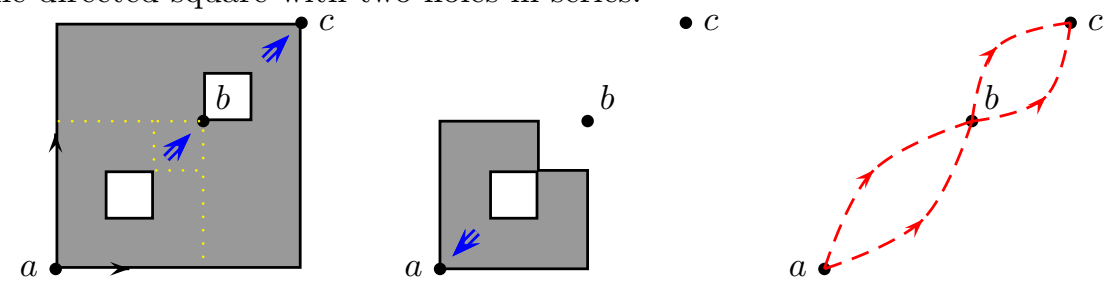

In all three cases we obtain a minimal extremal model. The first two are in fact equal to the fundamental bipartite graph. Notice that in the third example, we also have the branching information which is lost in the fundamental bipartite graph.

Example 1.13. Let $x \in \vec{S}^{1}$. The category $\vec{\pi}_{1}\left(\vec{S}^{1}, x\right)$ is isomorphic to the commutative monoid of non-negative integers under addition. For $y \in \vec{S}^{1}$, let $\left[\gamma_{y}\right]$ be the homotopy class of dipaths from $y$ to $x$ such that no proper subpath of $\gamma_{y}$ is a dipath from $y$ to $x$. This defines a future retract of $\vec{\pi}_{1}\left(\vec{S}^{1}\right)$. The induced functor

$$
P^{+}: \vec{\pi}_{1}\left(\vec{S}^{1}\right) \rightarrow \vec{\pi}_{1}\left(\vec{S}^{1}, x\right) \cong(\mathbb{N},+)
$$

is a minimal extremal model.

The simple proof of the following is in Section 3.

Proposition 1.14. An extremal model induces an injection of fundamental bipartite graphs.

We will see that if a d-space $X$ is a compact pospace, then this map is in fact an isomorphism (Theorem 3.3). 


\subsection{Directed van Kampen theorems}

One of the main tools for calculating the fundamental group and the fundamental groupoid is the Seifert-van Kampen theorem. A version of this theorem also applies to the fundamental category. It was proved by Goubault for local pospaces [14] and by Grandis for d-spaces [15]. These proofs follow R. Brown's proof of the usual van Kampen theorem for groupoids $[\mathbf{1}, \mathbf{2}, \mathbf{3}]$.

Let $X_{1}, X_{2} \subseteq X$ be d-spaces with $X$ equal to the union of the interiors of $X_{1}$ and $X_{2}$. Let $X_{0}=X_{1} \cap X_{2}$. Then

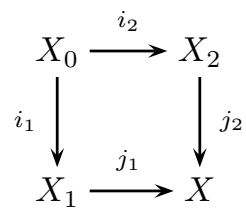

is a pushout in the category of d-spaces.

Theorem 1.15 ([15]). The induced commutative diagram

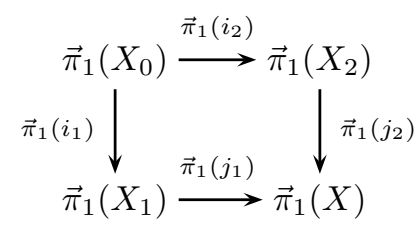

is a pushout in the category of small categories.

We prove one version of this theorem for full (co)reflective subcategories, and another for future retracts and past retracts. Let $X_{1}, X_{2} \subseteq X$ be d-spaces with $X$ equal to the union of the interiors of $X_{1}$ and $X_{2}$, and $X_{0}=X_{1} \cap X_{2}$. Let $A_{1}, A_{2} \subseteq A$ be d-spaces with $A=\operatorname{Int}\left(A_{1}\right) \cup \operatorname{Int}\left(A_{2}\right)$ and $A_{0}=A_{1} \cap A_{2}$. Assume that for $k=$ $1,2,3, A_{k} \subseteq X_{k}$. So, we have the following commutative diagram of d-spaces.

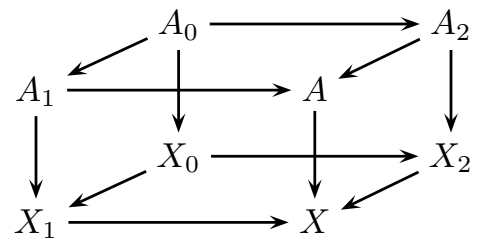

Theorem 1.16. Given compatible future retracts (solid arrows)

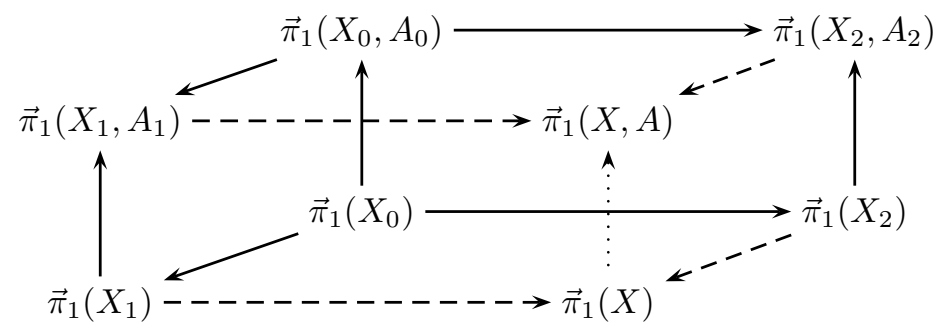

the top square, induced by (1.3), is a pushout of categories, and there is an induced retraction (dotted arrow) on the pushouts, which makes the diagram commute. 
The dual statement holds for past retracts.

We prove a more general version of Theorem 1.16 (Theorem 4.7), for triples $A \subseteq$ $B \subseteq X$. This allows us to apply the theorem inductively to obtain an analogous theorem for chains of compatible future retracts and past retracts (Theorem 4.9). We use this to obtain a van Kampen theorem for extremal models (Theorem 4.10). A simple application is given in Example 4.11.

\section{Preliminaries}

\subsection{Directed spaces and topological spaces}

We start by briefly relating directed spaces to topological spaces. Let Top and dTop denote the categories of topological spaces, and d-spaces, respectively.

Lemma 2.1. The underlying functor $U: \mathbf{d T o p} \rightarrow$ Top, given by $U(X, d X)=X$ and $U(f)=f$ has a left adjoint $F$ given by the constant paths. That is, $F(X)=(X, d X)$ where $d X$ is the set of constant paths and $F(f)=f$. The functor $U$ also has a right adjoint, $G$, given by all paths. That is, $G(X)=(X, d X)$ where $d X$ is the set of all (ordinary) paths in $X$, and $G(f)=f$.

Proof. The following are natural isomorphisms:

$$
\mathbf{d} \operatorname{Top}(F X,(Y, d Y)) \cong \boldsymbol{T o p}(X, Y)
$$

and

$$
\operatorname{Top}(X, Y) \cong \mathbf{d} \operatorname{Top}((X, d X), G Y)
$$

\subsection{Future retracts and past retracts}

For the convenience of the reader, we define past retracts explicitly.

Definition 2.2. A past retract of $\vec{\pi}_{1}(X)$ is a subspace $A \subseteq X$ together with a directed homotopy class of dipaths $\left[\gamma_{x}\right]$ with $\gamma_{x}(1)=x$ and $\gamma_{x}(0)=: x^{-} \in A$ such that for any $[\gamma]: a \rightarrow x$ with $a \in A$, there is a unique morphism making the following diagram commute.

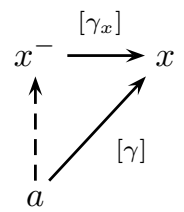

Again, we also insist that for $a \in A,\left[\gamma_{a}\right]=\left[\operatorname{Id}_{a}\right]$. We obtain a functor $P^{-}: \vec{\pi}_{1}(X) \rightarrow$ $\vec{\pi}_{1}(X, A)$, with $P^{-}\left(\vec{\pi}_{1}(\iota)\right)=\operatorname{Id}_{\vec{\pi}_{1}(X, A)}$.

Example 2.3. The following past retract is dual to the previous example of a future retract.

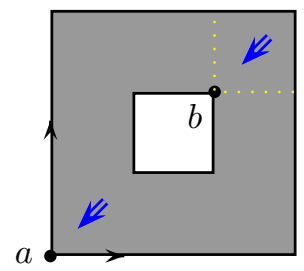


We now show that future retracts and past retracts have a succinct categorical definition. In fact, this is how they were first defined by Grandis [16] (who defined them for the fundamental category of a preordered space). Recall that a future retract induces a functor $P^{+}: \vec{\pi}_{1}(X) \rightarrow \vec{\pi}_{1}(X, A)$ with $P^{+}\left(\vec{\pi}_{1}(\iota)\right)=\operatorname{Id}_{\vec{\pi}_{1}(X, A)}$, and that a past retract induces a functor $P^{-}: \vec{\pi}_{1}(X) \rightarrow \vec{\pi}_{1}(X, A)$ with $P^{-}\left(\vec{\pi}_{1}(\iota)\right)=\operatorname{Id}_{\vec{\pi}_{1}(X, A)}$.

Proposition 2.4. There is a bijection between future retracts $\iota: A \subseteq X$ and adjunctions

$$
P^{+}: \vec{\pi}_{1}(X) \leftrightarrows \vec{\pi}_{1}(X, A): \vec{\pi}_{1}(\iota)
$$

with $P^{+}\left(\vec{\pi}_{1}(\iota)\right)=\operatorname{Id}_{\vec{\pi}_{1}(X, A)}$.

Proof. $(\Rightarrow)$ We have already shown that a future retract defines a functor

$$
P^{+}: \vec{\pi}_{1}(X) \rightarrow \vec{\pi}_{1}(X, A)
$$

with $P^{+}\left(\vec{\pi}_{1}(\iota)\right)=\operatorname{Id}_{\vec{\pi}_{1}(X, A)}$. The assignment $\eta_{x}: x \stackrel{\left[\gamma_{x}\right]}{\longrightarrow} x^{+}$is universal from $x$ to $\vec{\pi}_{1}(\iota)$, and determines a natural transformation $\eta: \operatorname{Id}_{\vec{\pi}_{1}(X)} \rightarrow \vec{\pi}_{1}(\iota) P^{+}$. Therefore $P^{+}$ is the left adjoint of $\vec{\pi}_{1}(\iota)$.

$(\Leftarrow)$ Assume we are given an adjunction $P^{+}: \vec{\pi}_{1}(X) \leftrightarrows \vec{\pi}_{1}(X, A): \vec{\pi}_{1}(\iota)$. For $x \in X$, the unit $\eta_{x}: x \rightarrow x^{+}$is universal from $x$ to $\vec{\pi}_{1}(\iota)$. That is, there is a unique morphism making the following diagram commute.

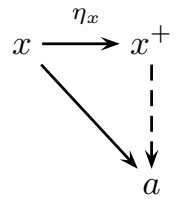

Since the inclusion of the full subcategory $\vec{\pi}_{1}(X, A)$ has a left adjoint, we say that $\vec{\pi}_{1}(X, A)$ is a full reflective subcategory of $\vec{\pi}_{1}(X)$. Dually, past retracts are equivalent to full coreflective subcategories.

Proposition 2.5. There is a bijection between past retracts $\iota: A \subseteq X$ and adjunctions

$$
\vec{\pi}_{1}(\iota): \vec{\pi}_{1}(X, A) \leftrightarrows \vec{\pi}_{1}(X): P^{-}
$$

with $P^{-}\left(\vec{\pi}_{1}(\iota)\right)=\operatorname{Id}_{\vec{\pi}_{1}(X, A)}$.

Remark 2.6. It follows that for future retracts and past retracts we have the following natural isomorphisms. For $x \in X$ and $a \in A$,

$$
\begin{aligned}
\vec{\pi}_{1}(X, A)\left(x^{+}, a\right) & \cong \vec{\pi}_{1}(X)(x, a), \\
\vec{\pi}_{1}(X)(a, x) & \cong \vec{\pi}_{1}(X, A)\left(a, x^{-}\right) .
\end{aligned}
$$

Generalizing Definitions 1.8 and 2.2 in the present language, we have:

Definition 2.7. A future (past) retract of $\vec{\pi}_{1}(X, A)$ is a full (co)reflective subcategory $\vec{\pi}_{1}(X, B)$, with $P^{ \pm}\left(\vec{\pi}_{1}(\iota)\right)=\operatorname{Id}_{\vec{\pi}_{1}(X, A)}$. 


\section{The fundamental bipartite graph}

Let $X$ have an extremal model: a chain of future retracts and past retracts

$$
\vec{\pi}_{1}(X) \stackrel{P_{1}^{+}}{\longrightarrow} \vec{\pi}_{1}\left(X, X_{1}\right) \stackrel{P_{2}^{-}}{\longrightarrow} \vec{\pi}_{1}\left(X, X_{2}\right) \stackrel{P_{3}^{+}}{\longrightarrow} \cdots \stackrel{P_{n}^{ \pm}}{\longrightarrow} \vec{\pi}_{1}(X, A),
$$

such that $\operatorname{Extrl}(X) \subseteq A$.

Proposition 3.1. An extremal model induces an injection of fundamental bipartite graphs.

Proof. By definition, $\operatorname{Extrl}\left(\vec{\pi}_{1}(X)\right) \subseteq A$. For $a \in A$, since $\vec{\pi}_{1}(X, A)$ is a subcategory of $\vec{\pi}_{1}(X)$, if $a \notin \operatorname{Extrl}\left(\vec{\pi}_{1}(X, A)\right)$ then $a \notin \operatorname{Extrl}\left(\vec{\pi}_{1}(X)\right)$. Combining these two facts we obtain that

$$
\operatorname{Extrl}\left(\vec{\pi}_{1}(X)\right) \subseteq \operatorname{Extrl}\left(\vec{\pi}_{1}(X, A)\right)
$$

Thus $\vec{\pi}_{1}\left(X, \operatorname{Extrl}\left(\vec{\pi}_{1}(X)\right)\right)$ is a subcategory of $\vec{\pi}_{1}\left(X, \operatorname{Extrl}\left(\vec{\pi}_{1}(X, A)\right)\right)$.

The map induced by future retracts and past retracts on the fundamental bipartite graph is not surjective in general. For example, take the unit interval $[0,1]$ and all (undirected) paths, and let $x \in[0,1]$. Then the map $[0,1] \rightarrow\{x\}$ induces a past and future retract. However $\operatorname{Extrl}([0,1])$ is empty while $\operatorname{Extrl}(\{x\})=\{x\}$.

We will show that if a d-space $X$ is a compact pospace, then this map is in fact an isomorphism.

Definition 3.2. A pospace is a topological space $X$, together with a reflexive, transitive, anti-symmetric relation $\leqslant$, such that $\leqslant$ is a closed subset of $X \times X$ in the product topology.

Given a d-space $X$, the fundamental category $\vec{\pi}_{1}(X)$ induces a preorder on $X$. Assume that this order makes $X$ into a compact pospace. Let

$$
\vec{\pi}_{1}(X) \stackrel{P_{1}^{+}}{\longrightarrow} \vec{\pi}_{1}\left(X, X_{1}\right) \stackrel{P_{2}^{-}}{\longrightarrow} \vec{\pi}_{1}\left(X, X_{2}\right) \stackrel{P_{3}^{+}}{\longrightarrow} \cdots \stackrel{P_{n}^{ \pm}}{\longrightarrow} \vec{\pi}_{1}(X, A)
$$

be an extremal model of $X$ in which for $1 \leqslant i \leqslant n, X_{i}$ is compact.

Theorem 3.3. Such an extremal model of a compact pospace induces an isomorphism of fundamental bipartite graphs.

Proof. Let $X$ be as above. Our proof is by induction on the number of retracts in the extremal model.

Let $P: \vec{\pi}_{1}(X) \rightarrow \vec{\pi}_{1}(X, B)$ be an extremal model as in (3.1) and let

$$
P^{+}: \vec{\pi}_{1}(X, B) \rightarrow \vec{\pi}_{1}(X, A)
$$

be a future retract. By Proposition 3.1, $P^{+} \circ P$ is injective on extremal points. We will show that $P^{+}: \operatorname{Extrl}\left(\vec{\pi}_{1}(X, B)\right) \rightarrow \operatorname{Extrl}\left(\vec{\pi}_{1}(X, A)\right)$. It will follow by induction that $P^{+} \circ P: \operatorname{Extrl}\left(\vec{\pi}_{1}(X)\right) \stackrel{\cong}{ } \operatorname{Extrl}\left(\vec{\pi}_{1}(X, A)\right)$.

Let $a$ be a maximal point in $\vec{\pi}_{1}(X, A)$. Let $b \in B$, with $a \leqslant b$. Then $a \leqslant b \leqslant b^{+}$. Since $b^{+} \in A$ and $a$ is maximal in $\vec{\pi}_{1}(X, A), a=b^{+}$. Since $\leqslant$is anti-symmetric, it follows that $a=b$. Therefore, $a$ is maximal in $\vec{\pi}_{1}(X, B)$. Thus the maximal points in $\vec{\pi}_{1}(X, A)$ are also maximal in $\vec{\pi}_{1}(X, B)$. 
Let $a$ be a minimal point in $\vec{\pi}_{1}(X, A)$. Since $A \subseteq B, a \in B$. By assumption $\vec{\pi}_{1}(X)$ induces an order $\leqslant$ on $X$ such that $X$ is a pospace. Order $B$ with the order induced by $\leqslant$. This coincides with the order induced by $\vec{\pi}_{1}(X, B)$. It is well known and easy to check that the induced order on a subspace of a pospace gives it the structure of a pospace. By assumption, $B$ is compact. Since $B$ is a compact pospace, there is a minimal point $b \in \vec{\pi}_{1}(X, B)$ such that $b \leqslant a\left[\mathbf{1 2}\right.$, Proposition VI-5.3], [22]. Since $P^{+}$ is a future retract, $b^{+} \leqslant a$. Since $a$ is minimal in $\vec{\pi}_{1}(X, A)$ and $b^{+} \in A$ it follows that $a=b^{+}$.

We remark that the compact condition is necessary. Consider $\mathbb{R}$ with $d \mathbb{R}$ all nondecreasing paths $[0,1] \rightarrow \mathbb{R}$. Then the induced order is the usual total order on $\mathbb{R}$ and it makes $\mathbb{R}$ into a pospace. There is a future retract $P^{+}$from $\mathbb{R}$ to the non-negative real numbers $\mathbb{R}_{\geqslant 0}$, where $x^{+}=x$ if $x \geqslant 0$ and $x^{+}=0$ if $x<0$. However $\operatorname{Extrl}(\mathbb{R})$ is empty while $\operatorname{Extrl}\left(\mathbb{R}_{\geqslant 0}\right)=\{0\}$.

\section{Directed van Kampen theorems}

We start this section by proving a version of the Seifert-van Kampen theorem for full subcategories of the fundamental category (Theorem 1.16). Our proof follows Grandis' proof of the van Kampen theorem for d-spaces [15], which in turn follows R. Brown's proof of the usual van Kampen theorem for groupoids $[\mathbf{1}, \mathbf{2}, \mathbf{3}]$. Instead of working with $A \subseteq X$ and the full subcategory $\vec{\pi}_{1}(X, A)$ of $\vec{\pi}_{1}(X)$, we work in the more general setting $A \subseteq B \subseteq X$ and the full subcategory $\vec{\pi}_{1}(X, A) \subseteq \vec{\pi}_{1}(X, B)$. The former can be obtained from the latter by setting $B=X$.

Next we prove a van Kampen theorem for past and future retracts. Finally we prove a van Kampen theorem for chains of past retracts and future retracts. As a corollary, we obtain a van Kampen theorem for extremal models.

Let $X_{1}, X_{2} \subseteq X$ be d-spaces with $X$ equal to the union of the interiors of $X_{1}$ and $X_{2}$. Let $X_{0}=X_{1} \cap X_{2}$. With these statements we assume that the d-space structure on $X$ is induced by the d-space structures on $X_{1}$ and $X_{2}$. That is, dipaths in $X$ are concatenations of dipaths in $X_{1}$ and $X_{2}$.

Similarly, let $A_{1}, A_{2} \subseteq A$ be d-spaces with $A=\operatorname{Int}\left(A_{1}\right) \cup \operatorname{Int}\left(A_{2}\right)$ and let $B_{1}, B_{2}$ $\subseteq B$ be d-spaces with $B=\operatorname{Int}\left(B_{1}\right) \cup \operatorname{Int}\left(B_{2}\right)$. Let $A_{0}=A_{1} \cap A_{2}$ and $B_{0}=B_{1} \cap B_{2}$. Assume that for $k=1,2,3, A_{k} \subseteq B_{k} \subseteq X_{k}$. Thus we have the following commutative diagram of d-spaces.

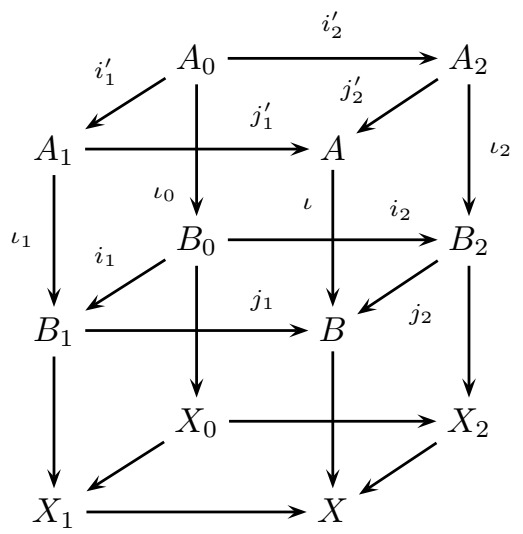


Furthermore, assume that $\vec{\pi}_{1}\left(X_{k}, A_{k}\right) \subseteq \vec{\pi}_{1}\left(X_{k}, B_{k}\right)$ is a full reflective subcategory for $k=0,1,2$ and that the following diagram commutes, where $P_{k}^{+}$denotes the reflections.

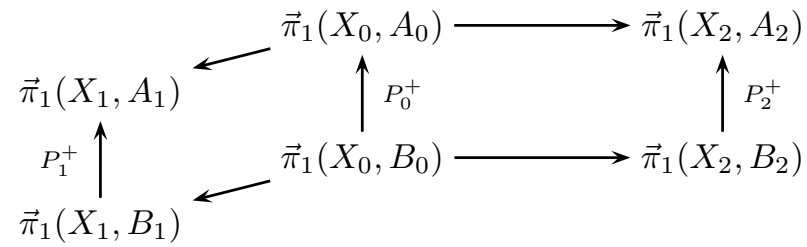

The following is our main lemma. We assume (4.1) and (4.2) with $B_{k}=X_{k}$ for $k=0,1,2$.

Lemma 4.1. Let $[\gamma] \in \vec{\pi}_{1}(X, A)$. Then there exist $\gamma_{1}, \ldots, \gamma_{n}$ with $\left[\gamma_{i}\right] \in \vec{\pi}_{1}\left(X_{1}, A_{1}\right)$ or $\vec{\pi}_{1}\left(X_{2}, A_{2}\right)$ such that $[\gamma]=\left[\gamma_{1}\right]+\cdots+\left[\gamma_{n}\right]$, where + denotes concatenation.

Proof. Let $[\gamma] \in \vec{\pi}_{1}(X, A)$ with $\gamma(0)=a$ and $\gamma(1)=a^{\prime}$. By the Lebesgue number lemma, there is a number $n$ such that $\gamma\left(\left[\frac{i-1}{n}, \frac{i}{n}\right]\right) \subseteq X_{k_{i}}$ where $k_{i} \in\{1,2\}$ for all $i=1, \ldots, n$. Let $x_{i}=\gamma\left(\frac{i}{n}\right), i=0, \ldots, n$. Let $\gamma_{i}: \vec{I} \rightarrow X$ be given by $\gamma_{i}(t)=\gamma\left(\frac{i-1+t}{n}\right)$. Then $\gamma=\gamma_{1}+\cdots+\gamma_{n}, \gamma_{i}: \vec{I} \rightarrow X_{k_{i}}$ and $\left[\gamma_{i}\right] \in \vec{\pi}_{1}\left(X_{k_{i}}\right)$. The only remaining problem is that we do not have $\left[\gamma_{i}\right] \in \vec{\pi}_{1}\left(X_{k_{i}}, A_{k_{i}}\right)$.

Let $\left[\gamma_{i}\right]^{+}$denote $P_{k_{i}}^{+}\left[\gamma_{i}\right]$. These maps of paths induce maps $x_{i} \mapsto x_{i}^{+}$which are well defined by the commutativity of (4.2). Composing the commutative diagrams

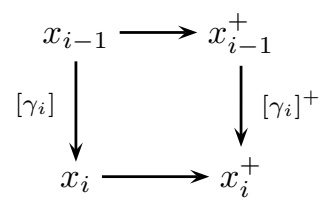

we obtain

$$
[\gamma]=\left[\gamma_{1}\right]+\cdots+\left[\gamma_{n}\right]=\left[\gamma_{1}\right]^{+}+\cdots+\left[\gamma_{n}\right]^{+},
$$

where $\left[\gamma_{i}\right]^{+} \in \vec{\pi}_{1}\left(X_{k_{i}}, A_{k_{i}}\right)$.

Theorem 4.2. The following diagram is a pushout of categories.

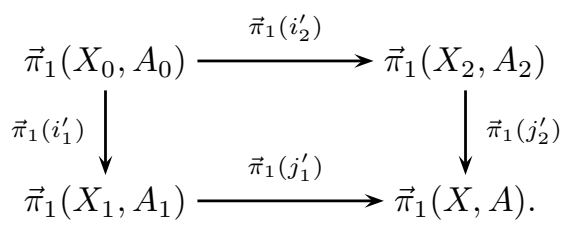

Proof. Let $\mathbf{C}$ be a category. Assume $\phi_{k}: \vec{\pi}_{1}\left(X_{k}, A_{k}\right) \rightarrow \mathbf{C}$ for $k=1,2$ such that $\phi_{1} \vec{\pi}_{1}\left(i_{1}^{\prime}\right)=\phi_{2} \vec{\pi}_{1}\left(i_{2}^{\prime}\right)$. Let $[\gamma] \in \vec{\pi}_{1}(X, A)$ with $\gamma(0)=a$ and $\gamma(1)=a^{\prime}$. Now we apply Lemma 4.1 to $[\gamma]$. Define $F[\gamma]=\phi_{k_{1}}\left[\gamma_{1}\right]+\cdots+\phi_{k_{n}}\left[\gamma_{n}\right]$, where addition is given by composition in $\mathbf{C}$.

We first remark that $F$ does not depend on the choice of $k_{i}$. If $\operatorname{im}\left(\gamma_{i}\right) \subset X_{1} \cap X_{2}=$ $X_{0}$, then the compatibility of $\phi_{1}$ and $\phi_{2}$ ensures that $\phi_{1}\left[\gamma_{i}\right]=\phi_{2}\left[\gamma_{i}\right]$. 
Next, $F$ does not depend on the choice of $n$ : given another suitable $m$, consider the partition into $\mathrm{nm}$ pieces.

Finally, $F$ does not depend on the choice of representative $\gamma$. Consider another $\tilde{\gamma} \simeq \gamma$. Again, apply Lebesgue's number lemma to $I \times I$ to suitably decompose the homotopy from $\gamma$ to $\tilde{\gamma}$ into homotopies contained in either $X_{1}$ or $X_{2}$. Now apply the suitable choice of $P_{1}^{+}$or $P_{2}^{+}$to each of these. Use the resulting set of homotopies in $\vec{\pi}_{1}\left(X_{1}, A_{1}\right)$ and $\vec{\pi}_{1}\left(X_{2}, A_{2}\right)$ to obtain

$$
F[\gamma]=F\left[\gamma_{1}\right]+\cdots+F\left[\gamma_{n}\right]+F\left[\operatorname{Id}_{a^{\prime}}\right]=F\left[\operatorname{Id}_{a}\right]+F\left[\tilde{\gamma}_{1}\right]+\cdots+F\left[\tilde{\gamma}_{n}\right]=F[\tilde{\gamma}] .
$$

Therefore $F$ is well defined.

For functoriality, notice that $F$ preserves compositions: if $\gamma, \gamma^{\prime}$ have decompositions

$$
\gamma=\gamma_{1}+\cdots+\gamma_{n} \text { and } \gamma^{\prime}=\gamma_{1}^{\prime}+\cdots+\gamma_{m}^{\prime},
$$

then $\gamma+\gamma^{\prime}$ has decomposition

$$
\gamma_{1}+\cdots+\gamma_{n}+\gamma_{1}^{\prime}+\cdots+\gamma_{m}^{\prime}
$$

The uniqueness of $F$ is by construction.

Lemma 4.3. Given the following commutative solid-arrowed diagram, let $F$ and $F^{\prime}$ be the pushout maps.

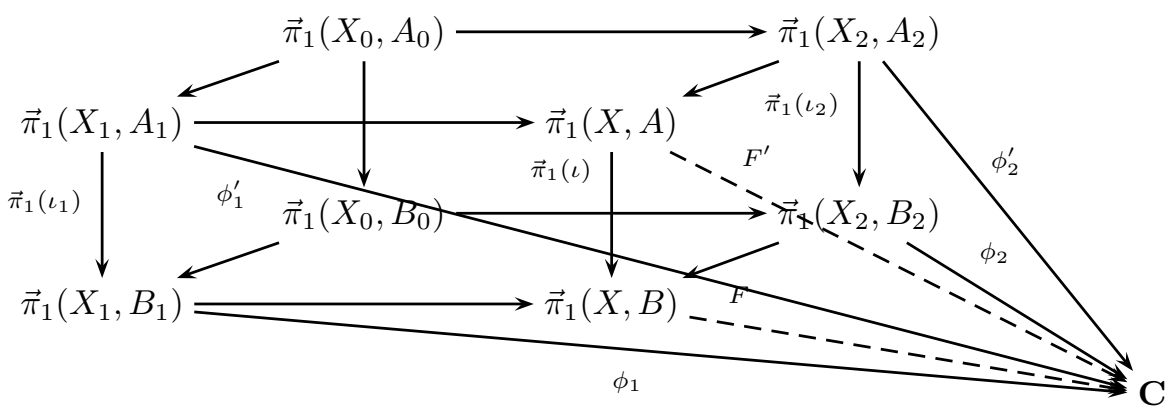

Then $F^{\prime}=F \vec{\pi}_{1}(\iota)$.

Proof. Let $[\gamma] \in \vec{\pi}_{1}(X, A)$. Apply Lemma 4.1 to $[\gamma]$.

$$
\begin{aligned}
F^{\prime}[\gamma] & =\phi_{k_{1}}^{\prime}\left[\gamma_{1}\right]+\cdots+\phi_{k_{n}}^{\prime}\left[\gamma_{n}\right] \\
& =\phi_{k_{1}} \vec{\pi}_{1}\left(\iota_{k_{1}}\right)\left[\gamma_{1}\right]+\cdots+\phi_{k_{n}} \vec{\pi}_{1}\left(\iota_{k_{n}}\right)\left[\gamma_{n}\right] \\
& =\phi_{k_{1}}\left[\gamma_{1}\right]+\cdots+\phi_{k_{n}}\left[\gamma_{n}\right] \\
& =F[\gamma] \\
& =F \vec{\pi}_{1}(\iota)[\gamma]
\end{aligned}
$$

Let Cat denote the category of categories. 
Theorem 4.4. The following diagram is a pushout in the arrow category on Cat.

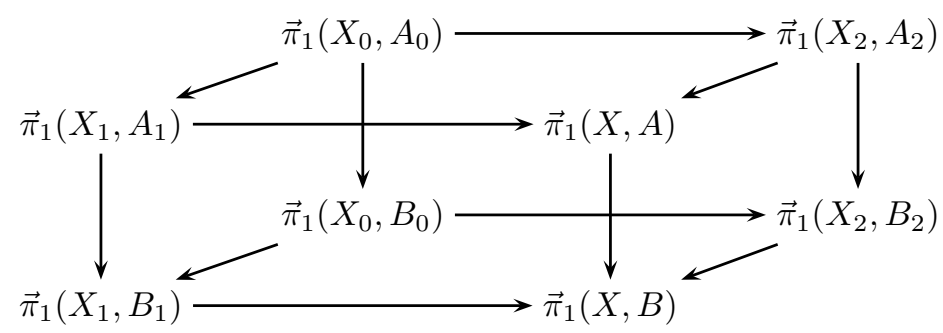

Proof. Let $F: \mathbf{C} \rightarrow \mathbf{D}$ be a functor between categories $\mathbf{C}$ and $\mathbf{D}$. We wish to show that given the solid-arrowed commutative diagram below, there are unique maps $G$ and $H$ making the diagram commute.

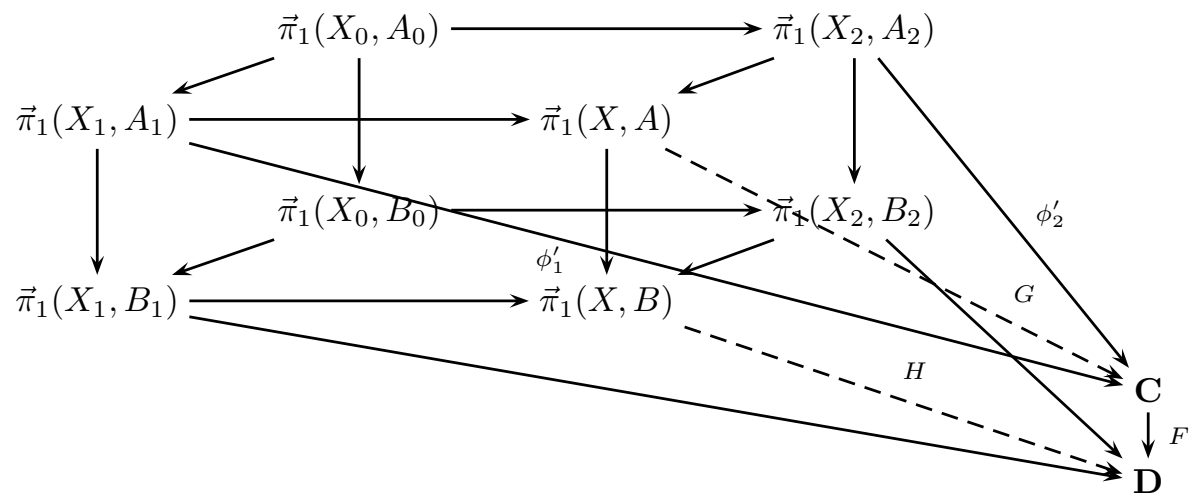

Since the top and bottom squares are pushouts, there are unique maps $G$ and $H$ making the top and bottom commute. For commutativity it remains to show that the following diagram commutes.

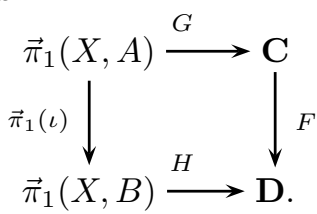

Since $F G$ is the pushout map of the following diagram

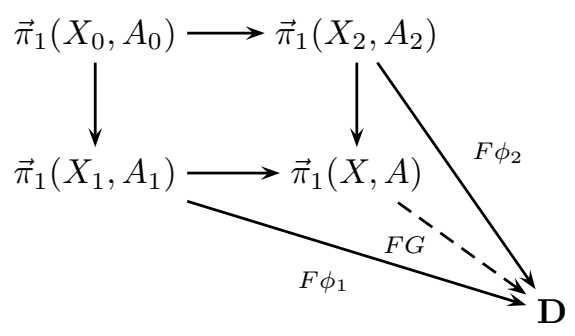

Lemma 4.3 tells us that $F G=H \vec{\pi}_{1}(\iota)$.

Finally, non-uniqueness of $(G, H)$ would contradict the uniqueness of $G$ and $H$. 
Given the commutative diagram (4.2) recall that $\vec{\pi}_{1}(X, B)$ and $\vec{\pi}_{1}(X, A)$ are the pushouts of the bottom two arrows and the top two arrows respectively. We will define a functor $P^{+}: \vec{\pi}_{1}(X, B) \rightarrow \vec{\pi}_{1}(X, A)$ and show that it is the pushout in the arrow category of Cat.

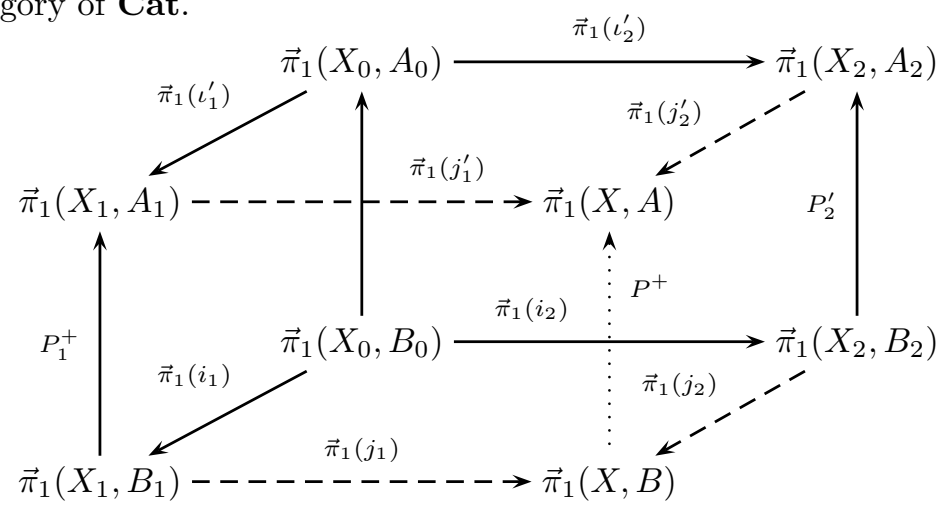

Definition 4.5. Define $P^{+}: \vec{\pi}_{1}(X, B) \rightarrow \vec{\pi}_{1}(X, A)$ as follows. For $x \in \vec{\pi}_{1}(X, B)$,

$$
P^{+}: x \mapsto \begin{cases}\vec{\pi}_{1}\left(j_{1}^{\prime}\right) P_{1}^{+} x & \text { if } x \in B_{1}, \\ \vec{\pi}_{1}\left(j_{2}^{\prime}\right) P_{2}^{+} x & \text { if } x \in B_{2} .\end{cases}
$$

Is this well defined? If $x \in B_{1} \cap B_{2}=B_{0}$, then they agree by the commutativity of the solid and dashed arrows in (4.3). Let $[\gamma] \in \vec{\pi}_{1}(X, B)$. By Lemma 4.1 there exist $\gamma_{1}, \ldots, \gamma_{n}$ with $\left[\gamma_{i}\right] \in \vec{\pi}_{1}\left(X_{k_{i}}, B_{k_{i}}\right)$ for $k_{i} \in\{1,2\}$ such that

$$
[\gamma]=\left[\gamma_{1}\right]+\cdots+\left[\gamma_{n}\right]
$$

Define

$$
P^{+}[\gamma]=\vec{\pi}_{1}\left(j_{k_{1}}^{\prime}\right) P_{k_{1}}^{+}\left[\gamma_{1}\right]+\cdots+\vec{\pi}_{1}\left(j_{k_{n}}^{\prime}\right) P_{k_{n}}^{+}\left[\gamma_{n}\right]
$$

This is well defined by the same argument as in the proof of Theorem 4.2. It will be convenient to denote $P^{+}(x)$ and $P^{+}[\gamma]$ by $x^{+}$and $[\gamma]^{+}$respectively.

Lemma 4.6. Let $[\gamma] \in \vec{\pi}_{1}(X, B)$ with $\gamma(0)=x, \gamma(1)=y$. Then the following diagram commutes.

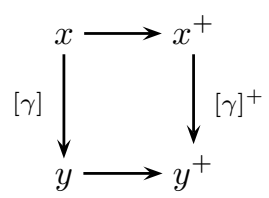

Proof. Let $\gamma_{1}, \ldots, \gamma_{n}$ be as in Definition 4.5. Let $\left[\gamma_{i}\right]^{+} \operatorname{denote} \vec{\pi}_{1}\left(j_{k_{i}}^{\prime}\right) P_{k_{i}}^{+}\left[\gamma_{i}\right]$. Composing the commutative diagrams

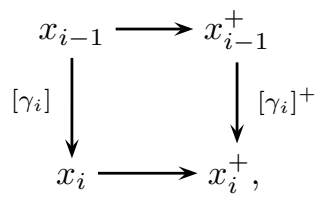

we obtain the desired result. 
Theorem 4.7. In (4.3), $P^{+}$is a pushout in the arrow category on Cat.

Proof. The theorem follows by the same argument as the one used to prove Theorem 4.4.

Theorem 4.8. Assume that $P_{k}^{+}$is the left adjoint of $\vec{\pi}_{1}\left(\iota_{k}\right)$ for $k=0,1,2$. Then there is an adjunction

$$
P^{+}: \vec{\pi}_{1}(X, B) \leftrightarrows \vec{\pi}_{1}(X, A): \vec{\pi}_{1}(\iota)
$$

Proof. The unit $\eta: 1_{\vec{\pi}_{1}(X, B)} \rightarrow \vec{\pi}_{1}(\iota) P^{+}$is a natural transformation by Lemma 4.6. The counit $\epsilon: P^{+} \vec{\pi}_{1}(\iota) \rightarrow 1_{\vec{\pi}_{1}(X, A)}$ is given by the identity. Finally, $\epsilon_{x^{+}} \circ P^{+}\left(\eta_{x^{+}}\right)=$ $\operatorname{Id}_{x+}$ and $\vec{\pi}_{1}(\iota)\left(\epsilon_{a}\right) \circ \eta_{a}=\operatorname{Id}_{a}$.

Assume that for $k=0,1,2$ we have chains of future retracts and past retracts

$$
\begin{aligned}
P_{k}: \vec{\pi}_{1}\left(X_{k, 0}\right) \stackrel{P_{k, 1}^{+}}{\longrightarrow} \vec{\pi}_{1}\left(X_{k, 0}, X_{k, 1}\right) & \\
\qquad \stackrel{P_{k, 2}^{-}}{\longrightarrow} & \vec{\pi}_{1}\left(X_{k, 0}, X_{k, 2}\right) \stackrel{P^{+} k, 3}{\longrightarrow} \cdots \rightarrow \vec{\pi}_{1}\left(X_{k, 0}, X_{k, n}\right)
\end{aligned}
$$

that are compatible. That is, for $\ell=1, \ldots, n, X_{1, \ell} \cap X_{2, \ell}=X_{0, \ell}, X_{\ell}=\operatorname{Int}\left(X_{1, \ell}\right) \cup$ $\operatorname{Int}\left(X_{2, \ell}\right)$, and the diagrams corresponding to $(4.2)$, but with $P_{0, \ell}, P_{1, \ell}$ and $P_{2, \ell}$, commute. Apply Theorem 4.2 to obtain pushouts $\vec{\pi}_{1}\left(X_{0}, X_{\ell}\right)$ for $\ell=1, \ldots, n$. Then use Definition 4.5 for each $\ell=1, \ldots, n$, to obtain

$$
P: \vec{\pi}_{1}\left(X_{0}\right) \stackrel{P_{1}^{+}}{\longrightarrow} \vec{\pi}_{1}\left(X_{0}, X_{1}\right) \stackrel{P_{2}^{-}}{\longrightarrow} \vec{\pi}_{1}\left(X_{0}, X_{2}\right) \stackrel{P_{3}^{+}}{\longrightarrow} \cdots \rightarrow \vec{\pi}_{1}\left(X_{0}, X_{n}\right) .
$$

Apply Theorem 4.7 inductively to obtain the following.

Theorem 4.9. The pushout of compatible chains of future retracts and past retracts is a chain of future retracts and past retracts.

It remains to apply this result to extremal models.

Theorem 4.10. The pushout of compatible compact extremal models is an extremal model.

Proof. It remains to show that if we have compatible extremal models, $P_{1}: \vec{\pi}_{1}\left(X_{1}\right) \rightarrow$ $\vec{\pi}_{1}\left(X_{1}, A_{1}\right)$ and $P_{2}: \vec{\pi}_{1}\left(X_{2}\right) \rightarrow \vec{\pi}_{1}\left(X_{2}, A_{2}\right)$, with $\operatorname{Extrl}\left(X_{1}\right) \subseteq A_{1}$ and $\operatorname{Extrl}\left(X_{2}\right) \subseteq A_{2}$, then the pushout $P: \vec{\pi}_{1}(X) \rightarrow \vec{\pi}_{1}(X, A)$ (Theorem 4.9) satisfies $\operatorname{Extrl}(X) \subseteq A$.

Let $x \in \operatorname{Extrl}(X)$, where $X=\operatorname{Int}\left(X_{2}\right) \cup \operatorname{Int}\left(X_{2}\right)$. Without loss of generality, we assume that $x \in X_{1}$. Then $x \in \operatorname{Extrl}\left(X_{1}\right)$ - otherwise this would contradict $x \in$ $\operatorname{Extrl}(X)$. Therefore, since $P_{1}$ is an extremal model, $x \in A_{1} \subseteq A$.

Example 4.11. Let $X_{1}$ be the subspace of $\vec{I} \times \vec{I}$ obtained by removing the two squares $(0.1,0.3) \times(0.4,0.6)$ and $(0.7,0.9) \times(0.4,0.6)$. Let $X_{2}$ be the d-space obtained by removing $(0.4,0.6) \times(0.4,0.6)$ from $\vec{I} \times \vec{I}$ and identifying $(0.2, y)$ and $(0.8, y)$ for $y \in$ $[0,1]$. Let $A_{1}=\{(0,0),(0.3,0),(0.5,0),(0.5,1),(0.7,1),(1,1)\}$ and let $A_{2}=\{(0,0)$, $(0.2,0)=(0.8,0),(0.2,1)=(0.8,1),(1,1)\}$. Let $X$ be obtained from $X_{1}$ and $X_{2}$ by identifying $[0.3,0.5] \times \vec{I}$ in $X_{1}$ with $[0,0.2] \times \vec{I}$ in $X_{2}$ and $[0.5,0.7] \times \vec{I}$ in $X_{1}$ with $[0.8,1] \times \vec{I}$ in $X_{2}$.

Let $P_{1,1}^{+}, P_{1,2}^{-}, P_{2,1}^{+}$, and $P_{2,2}^{-}$be the future retracts and past retracts of $X_{1}$ and $X_{2}$ indicated below. 

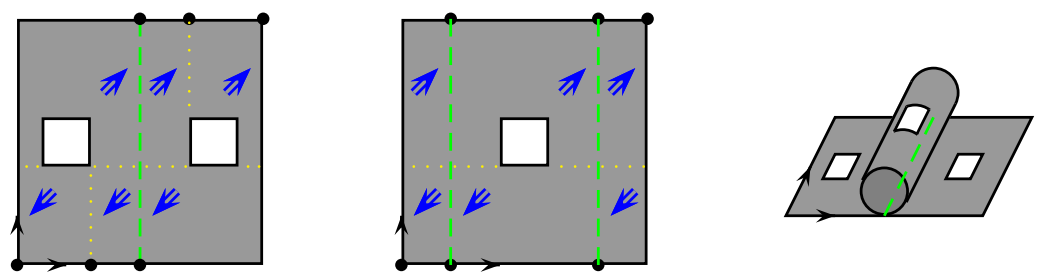

Then $\left(P_{1,1}^{+}, P_{1,2}^{-}\right)$and $\left(P_{2,1}^{+}, P_{2,2}^{-}\right)$are compatible extremal models. Then combining $\vec{\pi}_{1}\left(X_{1}, A_{1}\right)$ and $\vec{\pi}_{1}\left(X_{2}, A_{2}\right)$, we obtain $\vec{\pi}_{1}(X, A)$. These fundamental categories are generated by the graphs below.
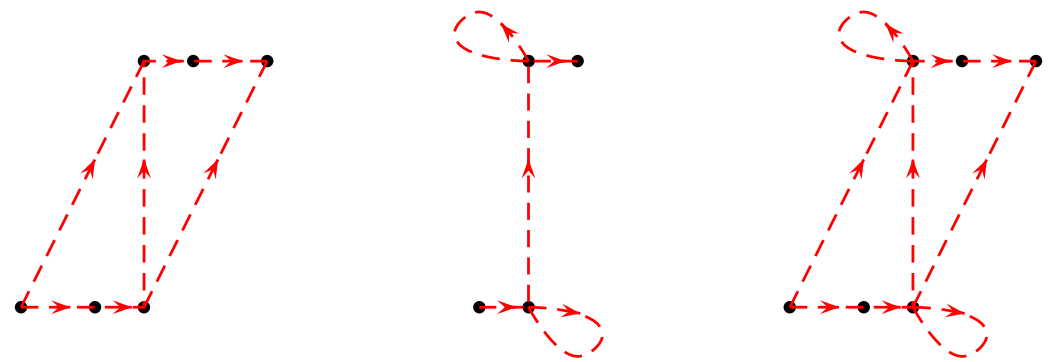

\section{References}

[1] R. Brown, Groupoids and van Kampen's theorem, Proc. London Math. Soc. (3) 17 (1967), 385-401.

[2] R. Brown, Topology and groupoids, Elements of modern topology, Third edition [McGraw-Hill, New York, 1968; MR0227979], BookSurge, LLC, Charleston, SC, 2006.

[3] R. Brown and A.R. Salleh, A van Kampen theorem for unions on nonconnected spaces, Arch. Math. (Basel) 42 (1984), no. 1, 85-88.

[4] P. Bubenik, Context for models of concurrency, Elect. Notes in Theor. Comp. Sci. 20 (2009), 3-21.

[5] P. Bubenik and K. Worytkiewicz, A model category for local po-spaces, Homology, Homotopy Appl. 8 (2006), no. 1, 263-292.

[6] L. Fajstrup, M. Raussen, E. Goubault, and E. Haucourt, Components of the fundamental category, Homotopy theory, Appl. Categ. Structures 12 (2004), no. 1, 81-108.

[7] L. Fajstrup, Dipaths and dihomotopies in a cubical complex, Adv. in Appl. Math. 35 (2005), no. 2, 188-206.

[8] L. Fajstrup, Cubical local partial orders on cubically subdivided spacesexistence and construction, Theoret. Comput. Sci. 365 (2006), no. 3, 199-205.

[9] L. Fajstrup, M. Raußen, and E. Goubault, Algebraic topology and concurrency, Theoret. Comput. Sci. 357 (2006), no. 1, 241-278.

[10] P. Gaucher, A model category for the homotopy theory of concurrency, Homology Homotopy Appl. 5 (2003), no. 5, 549-599. 
[11] P. Gaucher and E. Goubault, Topological deformation of higher dimensional automata, in Algebraic topological methods in computer science (Stanford, CA, 2001), Homology Homotopy Appl. 5 (2003), no. 2, 39-82 (electronic).

[12] G. Gierz, K.H. Hofmann, K. Keimel, J.D. Lawson, M. Mislove, and D.S. Scott, Continuous lattices and domains, Encyclopedia of Mathematics and its Applications 93, Cambridge University Press, Cambridge, 2003.

[13] E. Goubault and E. Haucourt, Components of the fundamental category. II, Appl. Categ. Structures 15 (2007), no. 4, 387-414.

[14] E. Goubault, Some geometric perspectives in concurrency theory, in Algebraic topological methods in computer science (Stanford, CA, 2001), Homology Homotopy Appl. 5 (2003), no. 2, 95-136 (electronic).

[15] M. Grandis, Directed homotopy theory. I, Cah. Topol. Géom. Différ. Catég. 44 (2003), no. 4, 281-316.

[16] M. Grandis, The shape of a category up to directed homotopy, Theory Appl. Categ. 15 (2005/2006), no. 4, 95-146 (electronic).

[17] M. Grandis, Quotient models of a category up to directed homotopy, Theory Appl. Categ. 16 (2006), no. 26, 709-735 (electronic).

[18] E. Haucourt, Categories of components and loop-free categories, Theory Appl. Categ. 16 (2006), no. 27, 736-770 (electronic).

[19] S. Krishnan, A convenient category of locally preordered spaces, arXiv:0808.1820 [math.AT], 2008.

[20] V. Pratt, Modeling concurrency with geometry, in Proceedings of the 18th ACM SIGPLAN-SIGACT symposium on principles of programming languages, pp. 311-322, ACM Press, 1991.

[21] M. Raussen, Invariants of directed spaces, Appl. Categ. Structures 15 (2007), no. $4,355-386$.

[22] A.D. Wallace, A fixed-point theorem, Bull. Amer. Math. Soc. 51 (1945), 413-416.

[23] K. Worytkiewicz, Some remarks on locally ordered spaces, arXiv: 0808.1820 [math.AT], 2008.

Peter Bubenik p.bubenik@csuohio.edu

http://academic.csuohio.edu/bubenik_p/

Department of Mathematics, Cleveland State University, 2121 Euclid Ave. RT 1515, Cleveland, OH 44115-2214, USA 